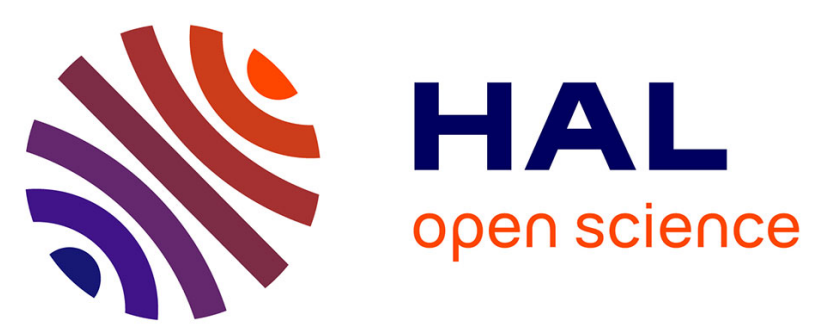

\title{
Stability of Bernstein-Greene-Kruskal plasma equilibria. Numerical experiments over a long time
}

A. Ghizzo, B. Izrar, Pierre Bertrand, E. Fijalkow, M. R. Feix, M. Shoucri

\section{To cite this version:}

A. Ghizzo, B. Izrar, Pierre Bertrand, E. Fijalkow, M. R. Feix, et al.. Stability of Bernstein-GreeneKruskal plasma equilibria. Numerical experiments over a long time. Physics of Fluids, 1988, 31 (1), pp.72-82. 10.1063/1.866579 . hal-01791844

\section{HAL Id: hal-01791844 \\ https://hal.univ-lorraine.fr/hal-01791844}

Submitted on 14 May 2018

HAL is a multi-disciplinary open access archive for the deposit and dissemination of scientific research documents, whether they are published or not. The documents may come from teaching and research institutions in France or abroad, or from public or private research centers.
L'archive ouverte pluridisciplinaire HAL, est destinée au dépôt et à la diffusion de documents scientifiques de niveau recherche, publiés ou non, émanant des établissements d'enseignement et de recherche français ou étrangers, des laboratoires publics ou privés. 


\section{Stability of Bernstein-Greene-Kruskal plasma equilibria. Numerical experiments over a long time}

A. Ghizzo, B. Izrar, P. Bertrand, E. Fijalkow, M. R. Feix, and M. Shoucri

Citation: The Physics of Fluids 31, 72 (1988); doi: 10.1063/1.866579

View online: https://doi.org/10.1063/1.866579

View Table of Contents: http://aip.scitation.org/toc/pfl/31/1

Published by the American Institute of Physics

\section{Articles you may be interested in}

Stability of Bernstein-Greene-Kruskal modes

Physics of Plasmas 7, 2425 (2000); 10.1063/1.874081

Electron holes in phase space: What they are and why they matter

Physics of Plasmas 24, 055601 (2017); 10.1063/1.4976854

Saturation process induced by vortex-merging in numerical Vlasov-Maxwell experiments of stimulated Raman backscattering

Physics of Plasmas 14, 072704 (2007); 10.1063/1.2749715

Solitary holes of negative energy and their possible role in the nonlinear destabilization of plasmas

Physics of Plasmas 9, 2462 (2002); 10.1063/1.1477450

Phase Space Hydrodynamics of Equivalent Nonlinear Systems: Experimental and Computational Observations The Physics of Fluids 13, 980 (1970); 10.1063/1.1693039

Stability of Bernstein-Greene-Kruskal equilibria

The Physics of Fluids 22, 1747 (1979); 10.1063/1.862811

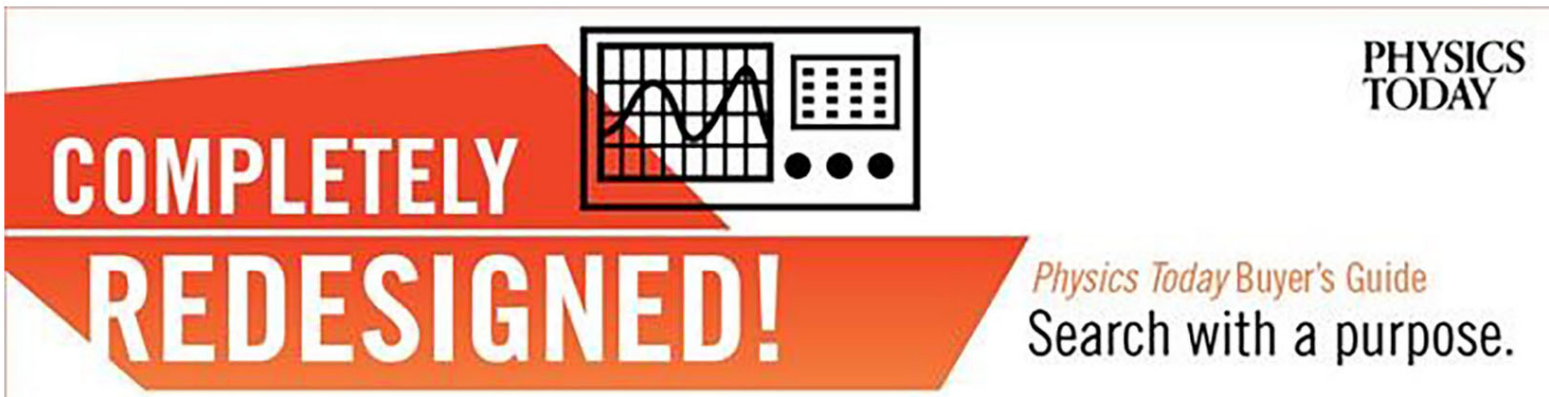




\title{
Stability of Bernstein-Greene-Kruskal plasma equilibria. Numerical experiments over a long time
}

\author{
A. Ghizzo, B. Izrar, and P. Bertrand \\ Physique Théorique, Université de Nancy I, Nancy, France \\ E. Fijalkow and M. R. Feix \\ PMMS/CNRS, Université d'Orléans, Orléans, France \\ M. Shoucri \\ Project Tokamak, IREQ Varennes, Québec, Canada
}

(Received 21 April 1987; accepted 8 September 1987)

\begin{abstract}
Bernstein-Greene-Kruskal (BGK) equilibria for a Vlasov plasma consisting of a periodic structure exhibiting depressions or "holes" in phase space are under consideration. Marginal stability analysis indicates that such structures are unstable when the system contains at least two holes. An Eulerian numerical code is developed allowing noiseless information on the long time phase space behavior (about $10^{3} \omega_{p}^{-1}$ ) to be obtained. Starting with equilibria with up to six holes, it is shown that the final state is given by a structure with only one large hole, the initial instability inducing coalescences of the different holes. On the other hand, starting with a homogeneous two-stream plasma it is shown that, in a first step, a BGK periodic structure appears with a number of holes proportional to the length of the system, followed, in a second step, by a coalescence of the holes to always end up with the above mentioned one large hole structure.
\end{abstract}

\section{INTRODUCTION}

It is well known that a one-dimensional, one species (with a fixed homogeneous neutralizing ion background) electron plasma can support spatially inhomogeneous equilibrium structures as shown by Bernstein, Greene, and Kruskal (BGK). ${ }^{1}$ Such an equilibrium is described by a distribution function $F(\epsilon)$ depending only on the energy of the particle

$$
\epsilon=\frac{1}{2} m v^{2}+e \phi,
$$

where $e$ is the electron charge and $m$ the electron mass. The stability of these structures has been extensively studied, but is not yet completely understood.

Using the useful water bag concept the stability has been shown for the monotonically decreasing function $F(\epsilon){ }^{2}$ However, in that case, no spatially periodic equilibria can be found; on the contrary, when $F(\epsilon)$ is not monotonically decreasing, especially when $F(\epsilon)$ is of a two-stream type, the plasma is able to support stationary large amplitude, spatially periodic structures exhibiting "holes" in phase space. Although all these structures are suspected to be unstable no general proof has yet been given, and all the solved examples exhibit instability. ${ }^{3-6}$

The available computer experiments on the topic ${ }^{6-9} \mathrm{did}$ not solve the question, mostly because of computer limitations. They deal with rather short systems during times of order $50-100 \omega_{p}^{-1}$, and suggest that the BGK structures could be the asymptotic state for homogeneous plasmas in the case of a two-stream instability. This fact, of course, would be in contradiction to the suspected general instability of the waves. In this paper, we will show that this "quasistabilization" is connected to the finite size of the system and that a system with only one hole is indeed stable. This result is in agreement with a theory predicting an evolution of ener- gy toward equilibrium structures of long wavelength ${ }^{10}$ for two-dimensional systems (either fluid or phase space).

The stability of a one-hole system is also consistent with a recent theory of Dupree ${ }^{11}$ showing that such a depression in the phase space density is a state of maximum entropy subject to constant mass, momentum, and energy, and is related to the physics of "clumps" that occur in a renormalized theory of weak turbulence. ${ }^{12}$ In addition, Ref. 11 contains entropy arguments explaining the tendency of holes to coalesce.

In Sec. II we consider the equilibrium properties of a BGK equilibrium of a two-stream type. The marginal stability analysis is investigated in Sec. III. The need to work over a long time period (up to $700 \omega_{p}^{-1}$ ) with a large spectrum of wavenumbers to obtain precise information on phase space points out the need for Eulerian codes. A new efficient code is presented in Sec. IV. The numerical experiments showing the coalescence of holes into a single one are then developed in Secs. V and VI, considering various types of initial conditions.

\section{TWO-STREAM BGK EQUILIBRIA}

The BGK structures are well-known stationary periodic solutions of the Vlasov-Poisson system, which, conveniently normalized, is written

$$
\begin{aligned}
& \frac{\partial f}{\partial t}+v \frac{\partial f}{\partial x}+E \frac{\partial f}{\partial v}=0, \\
& \frac{\partial E}{\partial x}=\int_{-\infty}^{\infty} f(x, v, t) d v-1
\end{aligned}
$$

$(x, v, t$ being, respectively, normalized to the Debye length, thermal velocity, and the inverse plasma frequency $\omega_{p}^{-1}$ ).

The stationary solution $(\partial / \partial t=0)$, being any function 
of the total energy $\epsilon=v^{2} / 2+\phi(x)$ of an electron, will be assumed throughout this paper to be in the form

$$
F(\epsilon)=\frac{\mu}{\sqrt{ } 2 \pi} \frac{2-2 \xi}{3-2 \xi}\left(1+\frac{\epsilon}{1-\xi}\right) \exp (-\epsilon) .
$$

In the homogeneous case $(\phi=0), F(\epsilon)$ corresponds to a two-stream plasma.

From (2) and (3) we deduce the electron density (normalized to the ion density)

$$
n(x)=\mu[(3-2 \xi+2 \phi) /(3-2 \xi)] \exp (-\phi) .
$$

Consequently, the equilibrium potential obeys the following equation:

$$
\frac{d^{2} \phi}{d x^{2}}+\mu \frac{3-2 \xi+2 \phi}{3-2 \xi} \exp (-\phi)-1=0 .
$$

Without loss of generality we can select the minimum of $n(x)$ at $x=0$ with $\phi(0)=0$ and $(d \phi / d x)(0)=0$. We have $n(0)=\mu$. The solution of Eq. (4) is obtained by a RungeKutta method and is characterized by the two parameters $\mu$ and $\xi$, where $\mu$ is the minimum value of the relative electron density and is a measure of the inhomogeneity of the system $(\mu \leqslant 1)$, and $\xi$ characterizes the depth of the hole in the phase space density around $x=0$. From (3) it is clear that $F$ is maximum at $\epsilon=\xi$. Then the ratio

$$
F(\epsilon=\xi) / F(\epsilon=0)=\exp (-\xi) /(1-\xi)
$$

is a measure of the depth of this hole centered around $x=0$ (with $\xi<1$ to ensure the positivity of $F$ ).

Equation (4) can also be written in terms of the electric field $E_{0}=-d \phi / d x$ :

$$
\frac{d^{2} E_{0}}{d x^{2}}-k^{2}(x) E_{0}=0,
$$

where

$$
k^{2}(x)=\mu[(1-2 \xi+2 \phi) /(3-2 \xi)] \exp (-\phi)
$$

This form is particularly important for the stability analysis. The numerical integration of (4) provides the potential $\phi(x)$, with a spatial period $\Lambda$. The electric field $E_{0}$, the trajectories in phase space, and the function $k^{2}(x)$ [given by (6) ] can then be easily deduced. Schematic forms are shown in Fig. 1.

To have periodic solutions for (4), $\xi$ and $\mu$ must obey some conditions. Let $\phi_{1}$ be the value of the potential at $x=x_{1}$ for which $k^{2}(x)$ vanishes. From (6) we deduce

$$
\phi_{1}=\xi-\frac{1}{2} \text {, }
$$

and consequently $\frac{1}{2}<\xi<1$. On the other hand, $d^{2} \phi / d x^{2}$, which is positive at $x=0$, must vanish at some point $x_{0}>0$ in order to obtain a periodic solution. From (4) and (7) the potential $\phi$ at this point must obey

$$
\left(1-\phi_{1}+\phi\right) \exp (-\phi)=\left(1-\phi_{1}\right) / \mu .
$$

Introducing an auxiliary parameter $\beta$ by the relation

$$
\left(1-\phi_{1}\right) / \mu=\beta \exp \left(-\phi_{1}\right),
$$

(8) becomes

$$
1+\phi-\phi_{1}=\beta \exp \left(\phi-\phi_{1}\right) .
$$

The elements of Eq. (10) as a function of $\phi$ are plotted in Fig. 2. A solution is possible only if $\beta<1$. Using (7) and (9) we

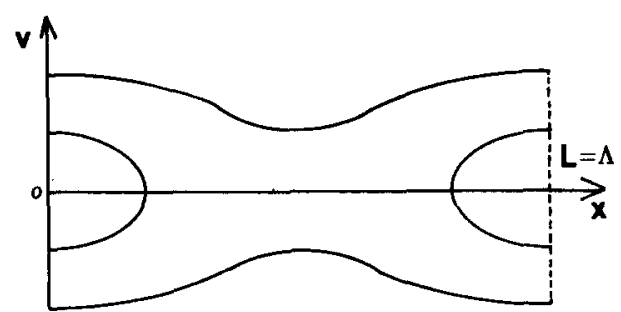

(a)

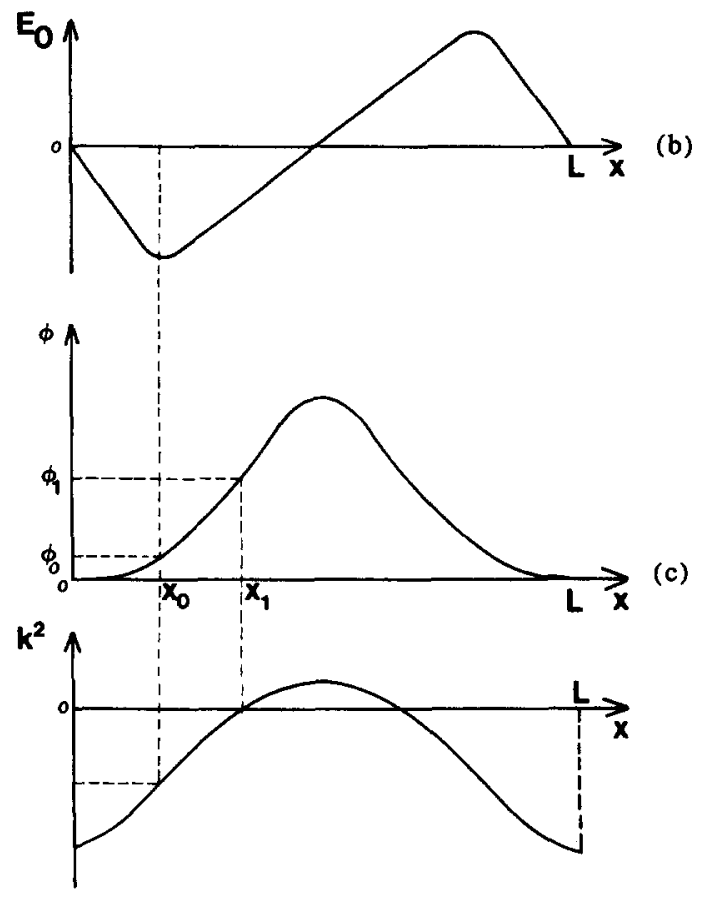

FIG. 1. Schematic plot of the distribution function in phase space (a), equilibrium electric field $E_{0}(\mathrm{~b})$, potential (c), and the function $k^{2}(x)$ as given by (6) for a one-period $(L=\Lambda)$ system. Here, $X_{0}$ is the point corresponding to $d^{2} \phi / d x^{2}=0$ (minimum of $E_{0}$ ) and $X_{1}$ is the point corresponding to $k^{2}(x)=0$.

obtain the following conditions between $\mu$ and $\xi$ :

$$
\mu>[(3-2 \xi) / 2] \exp \left(\xi-\frac{1}{2}\right),
$$

together with $\mu \leqslant 1$ and $\frac{1}{2} \leqslant \xi<1$. The region of the $\xi-\mu$ space,

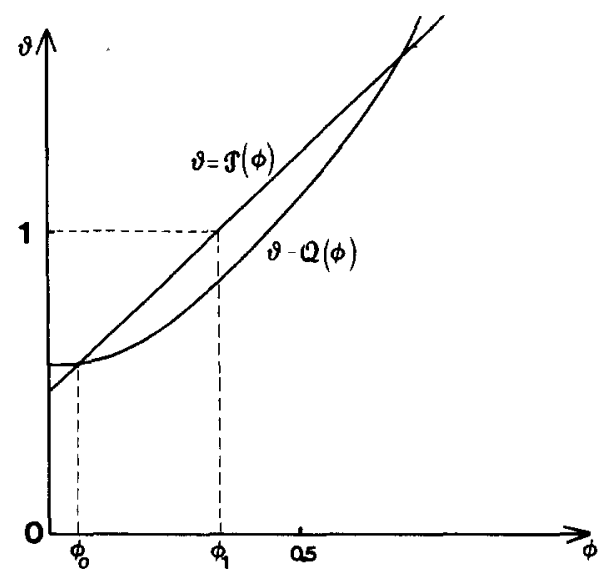

FIG. 2. Graphical solution of Eq. (10): $P(\phi)=Q(\phi)$, where $P(\phi)$ $=1+\phi-\phi_{1}$ and $Q(\phi)=\beta \exp \left(\phi-\phi_{1}\right)$. 


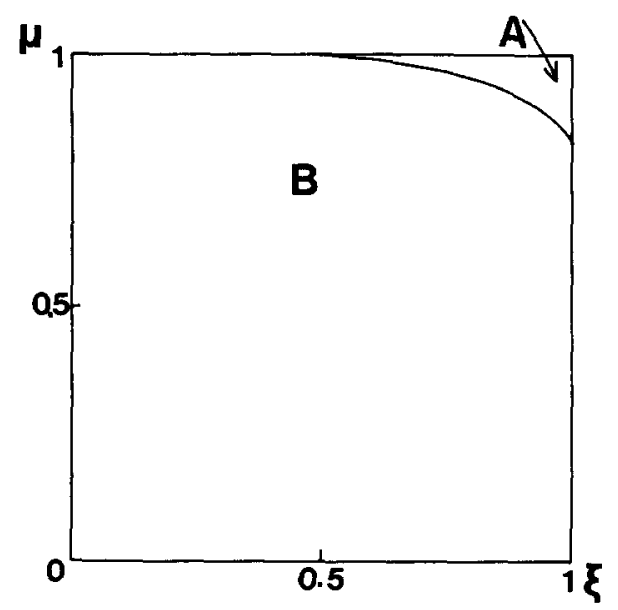

FIG. 3. A: Region of $\xi-\mu$ space corresponding to periodic BGK solutions. B: No periodic solution.

for which (4) or (5) have a periodic solution, is shown in Fig. 3.

Furthermore, it is interesting to point out that the value $\phi_{0}$ for which (10) is satisfied is such that $\phi_{0}<\phi_{1}$ [since from (9) we must have $\left.1-\phi_{1}<\beta \exp \left(-\phi_{1}\right)\right]$. Consequently,

$$
x_{0}<x_{1} .
$$

\section{MARGINAL STABILITY ANALYSIS}

Several methods have been used to study the stability of two-stream-type BGK structures, using mode coupling analysis, ${ }^{13}$ thermodynamic arguments, ${ }^{14}$ or computing the growth rate of normal modes. ${ }^{5,6}$ All these approaches predict the existence of instability.

Here we present a vastly different analysis, with the assumption that all instabilities are of the marginally unstable type just as Minardi did. ${ }^{14}$ We consider the stability of a wave train of $N$ periods (with one hole per period), each of length $\Lambda$ as defined in the preceding section, with a periodic boundary condition over the system of length $L=N \Lambda$. The originality of our approach is that it distinguishes between the case $N=1$ and $N \geqslant 2$. Some of the features have already been published. ${ }^{4-15}$ Here we outline especially the application to the two-stream-type BGK equilibria described in the preceding section.

Linearizing (1) and (2) around the BGK equilibrium,

$$
f=F+f_{1}, \quad E=E_{0}+E_{1},
$$

and supposing a time variation in $\exp i \omega t$, we obtain an equation for the perturbed potential $\psi=-\int E_{1}\left(x^{\prime}\right) d x^{\prime}$. We now suppose that the system becomes unstable through the point $\omega^{2}=0$. Such a mode (called the adiabatic marginal mode) is described by the differential equation

$$
\frac{d^{2} \psi}{d x^{2}}-k^{2}(x) \psi=0,
$$

where

$$
k^{2}(x)=-\int_{-\infty}^{+\infty} \frac{1}{v} \frac{\partial F}{\partial v} d v
$$

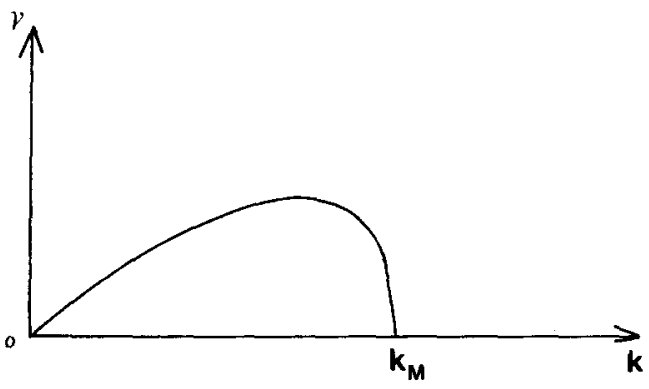

FIG. 4. Linear homogeneous two-stream instability growth rate as a function of the wavenumber $k$.

has the same meaning as (6). This equation has already been obtained by Santini. ${ }^{16}$

Let us first point out that for an infinite homogeneous plasma, $k^{2}$ as given by (14) is of course independent of $x$, and it is well known from the properties of the linear homogeneous dispersion function that the sign of $k^{2}$ indicates stability: $k^{2}>0$ for a stable plasma and $k^{2}<0$ for an unstable one. Furthermore $k_{M}^{2}=-k^{2}$ is just the square of the maximum wavenumber of instability (Fig. 4). In the inhomogeneous case $k^{2}$ is now dependent on $x$ and to obtain the stability criterion, we introduce the operator

$$
m=k^{2}(x)-\frac{d^{2}}{d x^{2}} .
$$

Let $\lambda$ be the eigenvalue of $m$ corresponding to the eigenfunction $\psi(\lambda)$. Note that this operator has at least the eigenvalue $\lambda=0$ since Eq. (13) is formally identical to the equilibrium equation (5). The eigenfunction $\psi(\lambda=0)$ is called the marginal mode. According to (13) the equation with $\lambda \neq 0$,

$$
m \psi(\lambda)=\lambda \psi(\lambda),
$$

corresponds to the marginal mode fitting a virtual plasma characterized by $k^{2}(x)-\lambda$ instead of $k^{2}(x)$. We have two possibilities.

(i) All the eigenvalues $\lambda$ are positive; then the virtual plasma is less "stable," and we must "destabilize" it in order to fit at least one marginal mode. We can conclude that the medium is stable.

(ii) At least one $\lambda$ is negative. For this eigenvalue, we stabilize the plasma to fit a marginal mode, and therefore we have instability.

Consequently, mathematically speaking, the stability property is connected to the computation of the eigenvalue of a Schrödinger-type operator, the sign of which indicates stability. For more details, see Ref. 15.

For a periodic plasma, the general way to solve (16) makes use of the Bloch-Floquet theorem and allows only a numerical computation of the eigenvalues. In order to obtain analytical results, it is interesting to consider another method, namely the Rayleigh coefficient one. For any function $G$ this coefficient, defined by

$$
\rho_{m}(G)=\int_{0}^{L} G m G d x\left(\int_{0}^{L} G^{2} d x\right)^{-1},
$$

is found to reach a minimal value if $G=\psi\left(\lambda_{\min }\right)$, where $\psi\left(\lambda_{\min }\right)$ is the eigenfunction of (16) corresponding to the 
smallest eigenvalue $\lambda_{\min }$ of (15). The minimal value of $\rho_{m}(G)$ is just $\lambda_{\min }$.

Thus if we are able to build a function $G$ such that $\rho_{m}(G)<0$, then $m$ has at least one negative eigenvalue, and the plasma is unstable. Furthermore, if a function $G$ can be associated with each equilibrium, this method provides a sufficient criterion of instability, irrespective of the proper- ties of the trial function $G$.

To construct the trial function $G$, we first note that the equilibrium field $E_{0}$ as given by ( 5 ) is a solution of $m E_{0}=0$ so that $\rho_{m}\left(E_{0}\right)=0$. We than build $G$ by modifying $E_{0}$ in the following way: we consider a system with two periods $(L=2 \Lambda)$ and we choose $G$ either equal to $\pm E_{0}(x)$ or the minimum or maximum of $E_{0}$, namely,

$$
G(x)= \begin{cases}E_{0}\left(x_{0}\right)=-E_{M}, & 0 \leqslant x \leqslant x_{0}, \\ E_{0}(x), & x_{0} \leqslant x \leqslant L / 2-x_{0}, \\ E_{0}\left(L / 2-x_{0}\right)=E_{m}, & L / 2-x_{0} \leqslant x \leqslant L / 2+x_{0}, \\ -E_{0}(x), & L / 2+x_{0} \leqslant x \leqslant L-x_{0}, \\ E_{0}(L)=-E_{M}, & L-x_{0} \leqslant x \leqslant L,\end{cases}
$$

where $\pm E_{m}$ are the maximum and minimum values of the equilibrium electric field (see Fig. 5). It is now obvious that $\rho_{m}(G)$ is given by

$$
\rho_{m}(G)=C \int_{0}^{x_{0}} k^{2}(x) d x,
$$

where $C$ is a positive constant. Since $x_{0}<x_{1}, k^{2}(x)$ is always negative between 0 and $x_{0}$ [Fig. 1(d)]. Thus $\rho_{m}(G)$ is negative proving the instability of a two-period system. Since our instability criterion is a sufficient one, the fact that the trial function is a single wavelength-type structure, although the equilibrium field $E_{0}$ has two wavelengths (see Fig. 5), is not important and $G$ must simply be continuous.

However, it must be pointed out that it is impossible to construct $G(x)$ by considering only one-period BGK equilibrium; $G$ would not be continuous, preventing the use of the Rayleigh coefficient method. However, such a construction can be extended to three or more periods (see Fig. 6 for a three-period BGK structure).

Thus we conclude that a sufficient condition of instability for a system containing $N$ periods is $N \geqslant 2$. Nothing definitive can be proved for $N=1$. Nevertheless, in the slightly inhomogeneous BGK equilibria it was noticed that the minimum wavenumber that can be fitted in the system, namely,

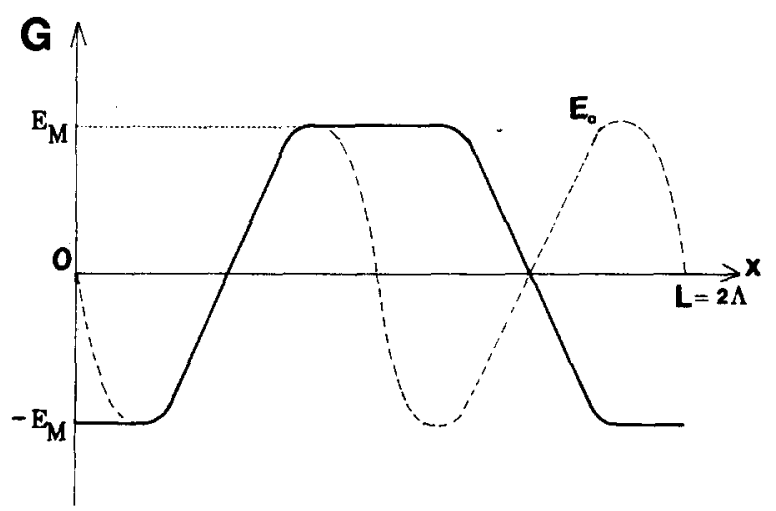

FIG. 5. Trial function $G$ for a two-period system. Here, $E_{0}$ is the equilibrium electric field.
$2 \pi / \Lambda$, is just equal to $k_{M}$, which is the maximum wavenumber for which the plasma is unstable. In that case, the equilibrium is at the marginal state. It can be conjectured that such limiting behavior will persist for strongly inhomogeneous plasma.

\section{THE NUMERICAL CODE}

Although particle codes are currently being used for strong nonlinear plasma turbulence, the finite number of particles that can be stored in the available computers leads to the appearance of numerical noise and to poor conservation of the main physical invariants. On the other hand, up to now, Eulerian codes have been successfully used only for weak nonlinear plasma and/or rather short time periods. However, the appearance of the vector computer points to the reactivation of such codes allowing better treatment of the phase space characteristics.

The best way to solve the Vlasov equation is to separate the integrations in both directions of the phase space plane. This is the well-known splitting scheme. ${ }^{17-19}$ We outline here some physical meanings of this method and several ways to improve it.

The crucial point is the representation of the electric field $E$ by a succession of Dirac pulses. Therefore, we have to solve the Vlasov equation (1) in which the electric field $E(x, t)$ has been replaced by $E^{*}(x, t)$ :

$$
E^{*}(x, t)=E(x, t) \Delta T \sum_{n} \delta\left(t-t_{n}\right),
$$

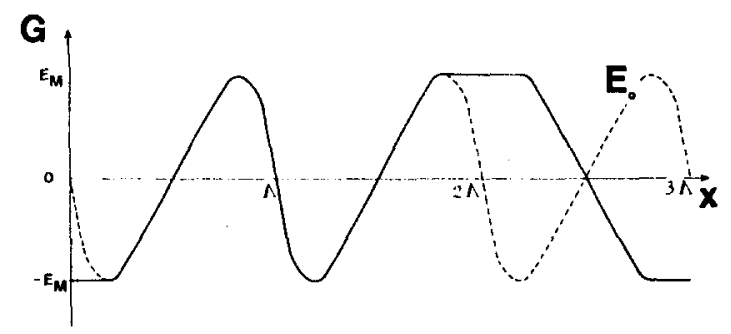

FIG. 6. The same as Fig. 5, but for a three-period system. 
where

$$
t_{n}=\left(n+\frac{1}{2}\right) \Delta T \text {. }
$$

Thus we obtain a new Vlasov system which will be called the Vlasov-Dirac combination (VDC) model throughout this paper. When the electric field is applied just at the middle of the time interval $\Delta T$, the exact integration is straightforward. Denoting $t_{n}^{-}$and $t_{n}^{+}$, respectively, as the time before and after the Dirac pulse, the time interval $n \Delta T$, $(n+1) \Delta T$ can be divided into three steps.

Step (a) $\left(n \Delta T \leqslant t<t_{n}^{-}\right)$: We have the free particle motion solution

$$
f\left(x, v, t_{n}^{-}\right)=f(x-v \Delta T / 2, v, n \Delta T),
$$

or performing a Fourier transform in the $x$ space ( $k$ being the conjugated position variable):

$$
f\left(k, v, t_{n}^{-}\right)=f(k, v, n \Delta T) \exp (-i k v \Delta T / 2) .
$$

Step $(b)\left(t=t_{n}\right):$ We apply the Dirac pulse and get the formal solution

$$
f\left(x, v, t_{n}^{+}\right)=f\left[x, v-E\left(x, t_{n}^{-}\right) \Delta T, t_{n}^{-}\right],
$$

or performing a Fourier transform of the $v$ space ( $\lambda$ being the conjugated velocity variable):

$$
f\left(x, \lambda, t_{n}^{+}\right)=f\left(x, \lambda, t_{n}^{-}\right) \exp \left[-i \lambda E\left(x, t_{n}^{-}\right) \Delta T\right] .
$$

It is obvious that $f\left(x, \lambda=0, t_{n}^{+}\right)=f\left(x, \lambda=0, t_{n}\right)$ so that $E\left(x, t_{n}^{+}\right)=E\left(x, t_{x}^{-}\right)$. The electric field appearing in (18) has to be computed from the Poisson equation (2) only at $t=t_{n}^{-}$, i.e., at the end of step (a).

Step (c) $\left(t_{n}^{+}<t \leqslant(n+1) \Delta T\right)$ : We again perform step (a) starting with $f\left(x, v, t_{n}^{+}\right)$.

It must be pointed out that Eqs. (19) and (20) give an exact solution of the VDC equation. From a numerical point of view it has been shown that this solution provides a second order scheme for the Vlasov equation. ${ }^{17}$ However, from a physical point of view, it is very important to understand how the VDC model incorporates some basic plasma properties such as the linear theory. To achieve this, let us linearize $f$ around an homogeneous equilibrium

$$
f(x, v, t)=F_{0}(v)+f_{1}(x, v, t) .
$$

After performing the usual Laplace and Fourier transforms, the linearized VDC equations (1)-(18) yield

$$
\begin{aligned}
&-i k {\left[E(k, s)+\frac{i}{k} \sum E\left(k, s-\frac{2 \pi i m}{\Delta T}\right)\right.} \\
&\left.\quad \times \int_{-\infty}^{\infty} \frac{d F_{0} / d v}{s-i k v} d v\right]=\int_{-\infty}^{\infty} \frac{f_{1}(k, v, 0)}{s-i k v} d v .
\end{aligned}
$$

However, the appearance of a sum on the lhs of (21) does not allow us to recover directly the plasma dispersion function $\epsilon(k, s)$. Let us introduce the Shannon theorem: if the frequency spectrum of $E(k)$ is restricted to an interval $\left(-\omega_{M},+\omega_{M}\right)$, we must have

$$
\Delta T \leqslant \pi / \omega_{M} \text {. }
$$

If this condition is fulfilled, the sum on the lhs (21) gives a nonzero contribution only for $m=0$, allowing us to recover the usual dispersion function. Since we know that the frequency spectrum of the electric field does not exceed a few $\omega_{p}$, we see that a choice of $\Delta T$, such that $\omega_{p} \Delta T \ll 1$, is enough to take fully into account the field time variation.

Finally the remaining problem is to solve (19) and (20), i.e., compute $f((n+1) \Delta T)$ from $f(n \Delta T)$. A powerful method is to effect the successive shift in (19a) and (20a) using cubic spline interpolation. ${ }^{19}$ On the other hand, the fact that the shifts, respectively, in $x$ and $v$ space, can be expressed in the Fourier space by simply changing the phase [see, respectively, (19b) or (20b)] suggests the use of the fast Fourier transform (FFT), which can be efficiently run on the vector computer. Both methods run roughly like $N$, where $N$ is the total number of points. Then the successive shifts are achieved in the following way: (a) free particle

\section{PLASMA DISTRIBUTION FUNCTION}
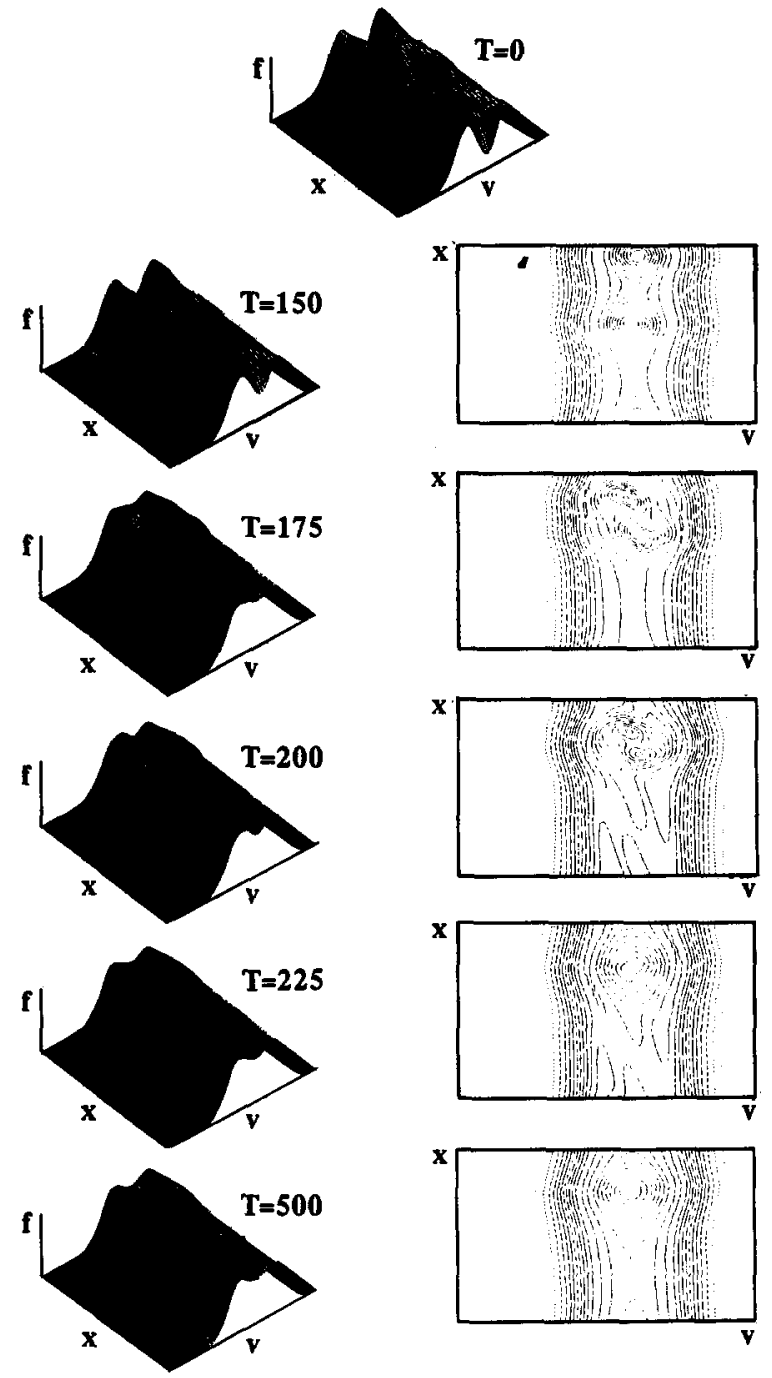

FIG. 7. Contour plot and 3-D curves showing the evolution $(T=0,150$, $175,200,225$, and final state at $T=500, T$ standing for $\omega_{p} t$ ) of a slightly perturbed BGK equilibrium that originally had two holes. Note the rather "clean" structure of the final state. 
motion from $n \Delta T$ to $t_{n}$,

$$
\begin{aligned}
f(x, v, n \Delta T) & \stackrel{F T x}{\rightarrow} f(k, v, n \Delta T) \stackrel{x}{\rightarrow} \exp (-i k v \Delta T / 2) \\
& \stackrel{I F T x}{\rightarrow} f\left(x, v, t_{n}^{-}\right) ;
\end{aligned}
$$

(b) Dirac pulse at $t=t_{n}$,

$$
\begin{aligned}
f\left(x, v, t_{n}^{-}\right) & \stackrel{F T v}{\rightarrow} f\left(x, \lambda, t_{n}^{-}\right) \\
& x \\
& \rightarrow \exp \left[-i \lambda E\left(x, t_{n}^{-}\right) \Delta T\right]^{I F T v} \rightarrow f\left(x, v, t_{n}^{+}\right) ;
\end{aligned}
$$

(c) again we perform step (a) from $t_{n}^{+}$to $(n+1) \Delta T$.

Here, $F T x$, respectively, $F T$, means Fourier transform with respect to $x$, respectively, IFT, means inverse Fourier transform, and $\times$ means multiplication (i.e., shifting the phase). At the end of step (a), the electric field is computed by solving the Poisson equation through Fourier transform.

This code is extremely stable and a time step of $\omega_{p}$ $\Delta T=0.25$ can be used with no noticeable difference with a five times smaller time step. To avoid aliasing (which results from the nonperiodic character of velocity space), a large number of values of the distribution function above the cutoff $\left|v_{\max }\right|=6$ up to $|v|=18$ is set equal to zero. As a consequence, typically, among 512 points, only 178 are useful. In the $x$ space, 128 or 256 points are needed, depending on the box length.

Although the experiments described in this paper have been performed with the Fourier code, an excellent agreement has been obtained with the cubic spline code of Shoucri and Gagné. ${ }^{19}$

\section{EVOLUTION OF AN INITIAL NHOLE BGK STRUCTURE}

We start with a structure as described by Eqs. (3) and (4). In all the runs we select $\xi=0.90$ and $\mu=0.92$ in the region of the $\xi-\mu$ space where (4) has a periodic solution (Fig. 3). The spatial period $\Lambda=14.710$ corresponds to a single vortex structure. An $N$ vortex structure is constructed by adding $N$ periods (here $N$ varies between 1 and 6 ). This structure is slightly perturbed at time $t=0$ with

$$
f(x, v, 0)=F(\epsilon)\left[1+\alpha \cos \left(k_{0} x+\psi\right)\right],
$$
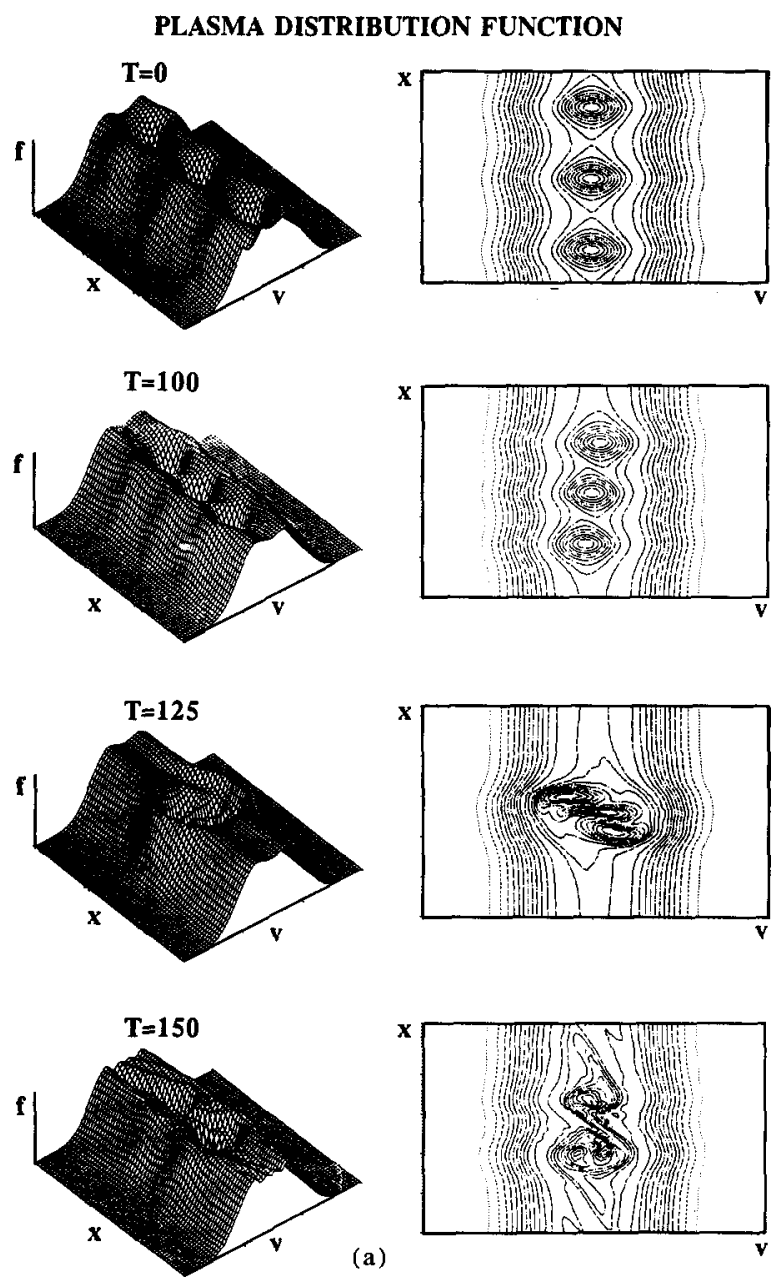

\section{PLASMA DISTRIBUTION FUNCTION}
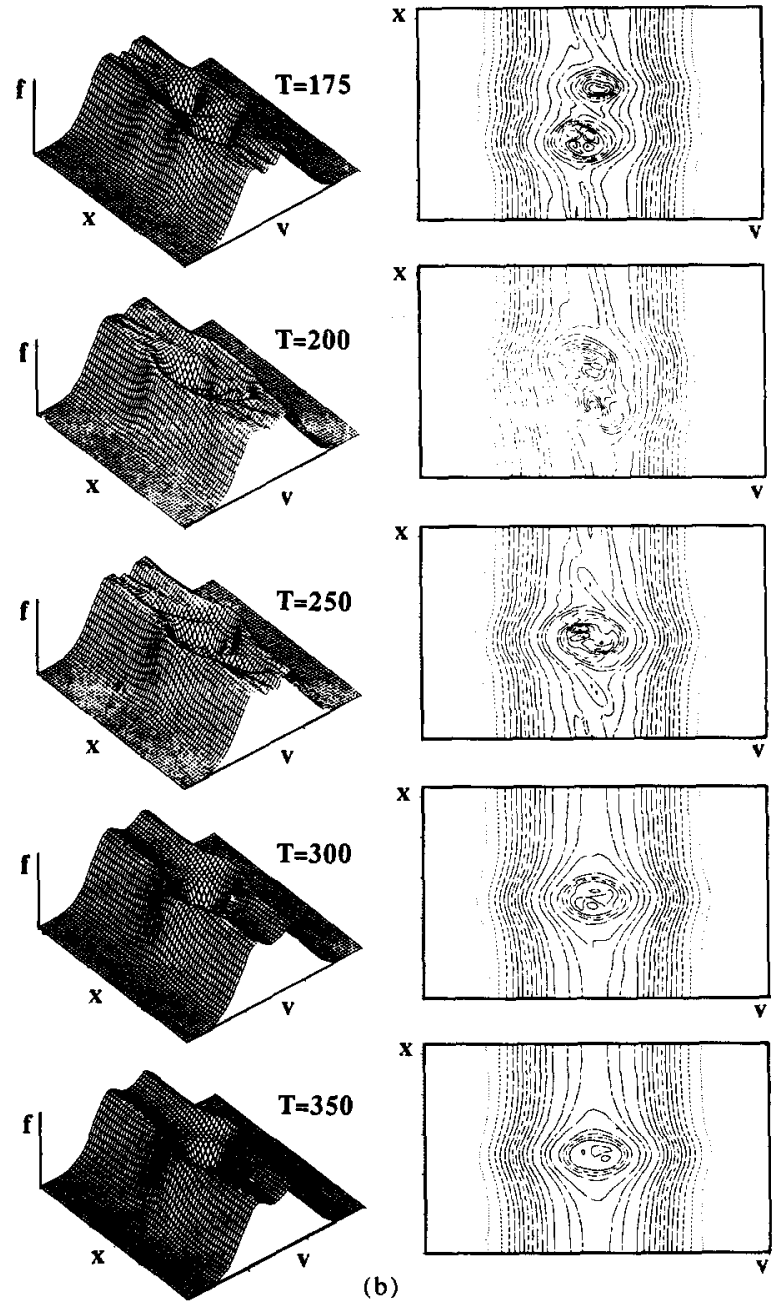

(b)

FIG. 8. The same as Fig. 7, for a three-hole initial condition. Note the "cleaning" of the microstructure of the final state between 250 and $350 \omega_{p}^{-1}$. 

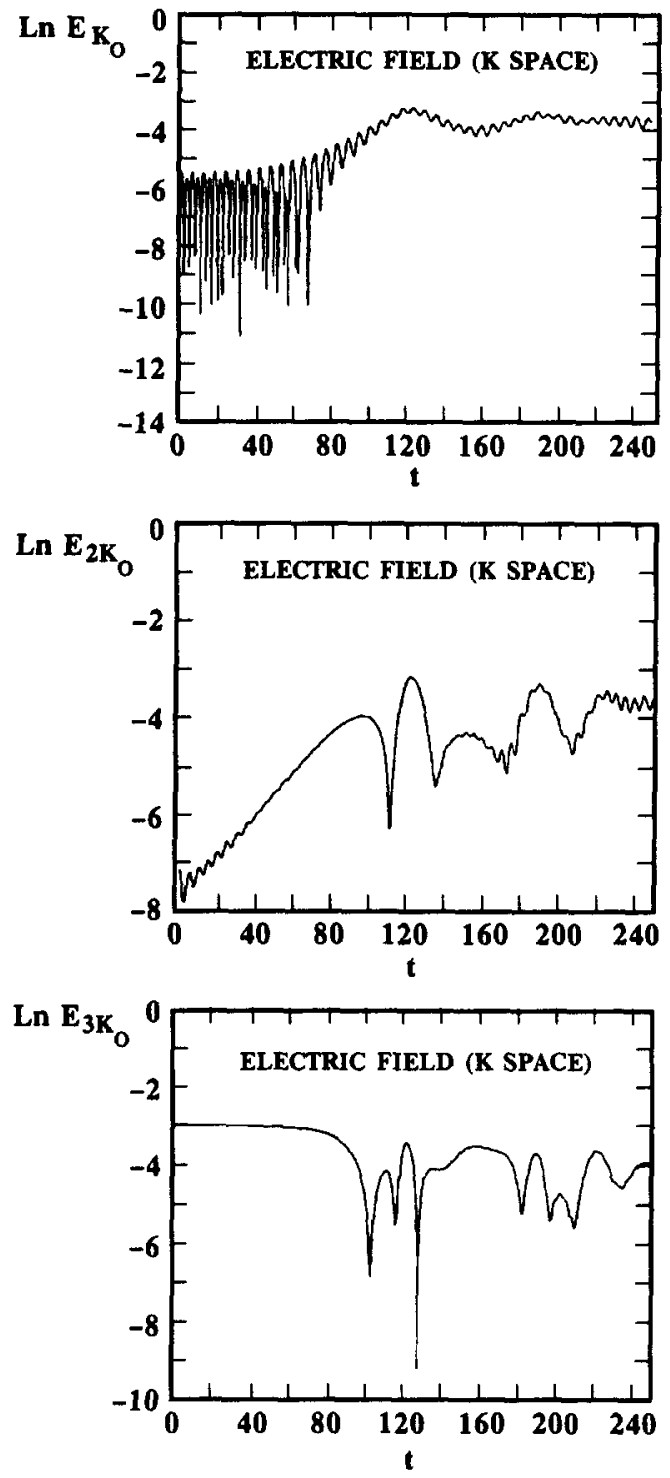

FIG. 9. Evolution of the first three modes of the electric field as a function of time, for the three-hole initial condition (case of Fig. 8).

$k_{0}=2 \pi / L$, where $L=N \Lambda$ is the total length of the system, $\psi$ is a phase shift allowing a breaking of the initial symmetry and consequently an acceleration of the evolution, and $\alpha$ is typically $10^{-3}-10^{-4}$.

The first series of experiments was conducted with $N=1$ showing no evolution up to $\omega_{p} t=500$ and consequently strengthening the conjecture of a marginally stable structure. Next, we consider the case $N=2$ (see Fig. 7 for $\omega_{p} t=0$ showing the three-dimensional plot of the initial condition). The further curves represent, at different times, the 3-D plot of the distribution function $f(x, v)$ and the corresponding $f=c^{t}$ contours. The initial configuration evolves slowly (nothing happens before $\omega_{p} t=100$ ), but at $\omega_{p} t=150$ the two holes start coalescing. The lack of symmetry is connected to the nonzero value of the initial phase shift $\psi$ in (22). The fusion of the two holes is achieved at $\omega_{p} t=200$ and only one hole remains. An interesting result is the rather smooth structure in the final state in phase space $\left(\omega_{p} t=500\right)$.

Let us now consider the case $N=3$ (Fig. 8). Again, three different steps can be noticed. In the first step, the holes begin to move through a mutual attraction. Next they coalesce, and we are left with a final equilibrium state, which consists again of a single smooth hole in which the noisy microstructure disappears (from $\omega_{p} t=250$ to $\omega_{p} t=350$ ). The temporal behavior of the first three modes of the electric field $\left(E_{k_{0}}, E_{2 k_{0}}, E_{3 k_{0}}\right)$ is shown in Fig. 9. Modes $k_{0}$ and $2 k_{0}$ exhibit the usual unstable behavior from $t=0$ to $t=100$ $\omega_{p} t$, and the further nonlinear saturation of the instability corresponding to the coalescence of the three vortices. We feel that while a one-hole structure is at the stability limit, mode $3 k_{0}$ is the marginal one and indeed remains constant before coalescence.

The $N=4$ case has been presented in an earlier paper, ${ }^{20}$ and is shown again in Fig. 10. Because $\psi \neq 0$, the coalescence of the three central holes is not perfectly symmetric, and one hole is expelled ( $\left.\omega_{p} t=175-200\right)$. This hole collides with the one above it, with a final fusion of the two "superholes" and the formation of a stable smooth one-hole structure after a time of order $500 \omega_{p}^{-1}$.

Finally we have considered the case $N=6$ (Fig. 11). After colliding by pairs, we are left at $\omega_{p} t=200$ with three "superholes" with a further evolution similar to the $N=3$ case, and again, a final single hole in which the noisy microstructure disappears $\left(500-700 \omega_{p} t\right)$.

Consequently, the BGK structure appears to be unstable (except the one-hole structure), and the claim that they can be the asymptotic state of two-stream instability seems to contradict the above results.

\section{EVOLUTION OF A HOMOGENEOUS TWO-STREAM PLASMA}

In the earlier numerical experiments, ${ }^{8,9}$ because of time and memory computer limitations, rather short systems were, in fact, considered. It is therefore tempting to consider again this problem with a much longer plasma.

Thus we take $F(\epsilon)$ with $\mu=1, \phi=0$ (homogeneous case). The marginal mode (i.e., the maximum wavenumber of instability) is given by Eq. (6), which yields in that case

$$
k_{M}^{2}=-k^{2}=(2 \xi-1) /(3-2 \xi) .
$$

Choosing $\xi=0.90$, we have $k_{M}=0.816$, with a marginal wavelength of $\lambda=7.69$. This two-stream plasma, shown in Fig. 12, is slightly perturbed at $t=0$ on the fundamental mode $k_{0}=2 \pi / L$, where $L=30.78$ is the length of the plasma and is four times the marginal wavelength. Consequently, we forecast the appearance of a two-hole structure, which, indeed, shows up rather quickly $\left(\omega_{p} t=40\right)$.

Since it is no more than an approximate equilibrium, the evolution is much faster than the $N=2$ case of Sec. V, and the two holes merge rapidly. At time $t=100 \omega_{p}^{-1}$, the large central hole is quite apparent, with the damping of the small wavelength structure nearly completed at $t=160 \omega_{p}^{-1}$.

Other computer experiments with still longer systems have been performed exhibiting the same phenomena, i.e., first the appearance of a number of holes proportional to the 

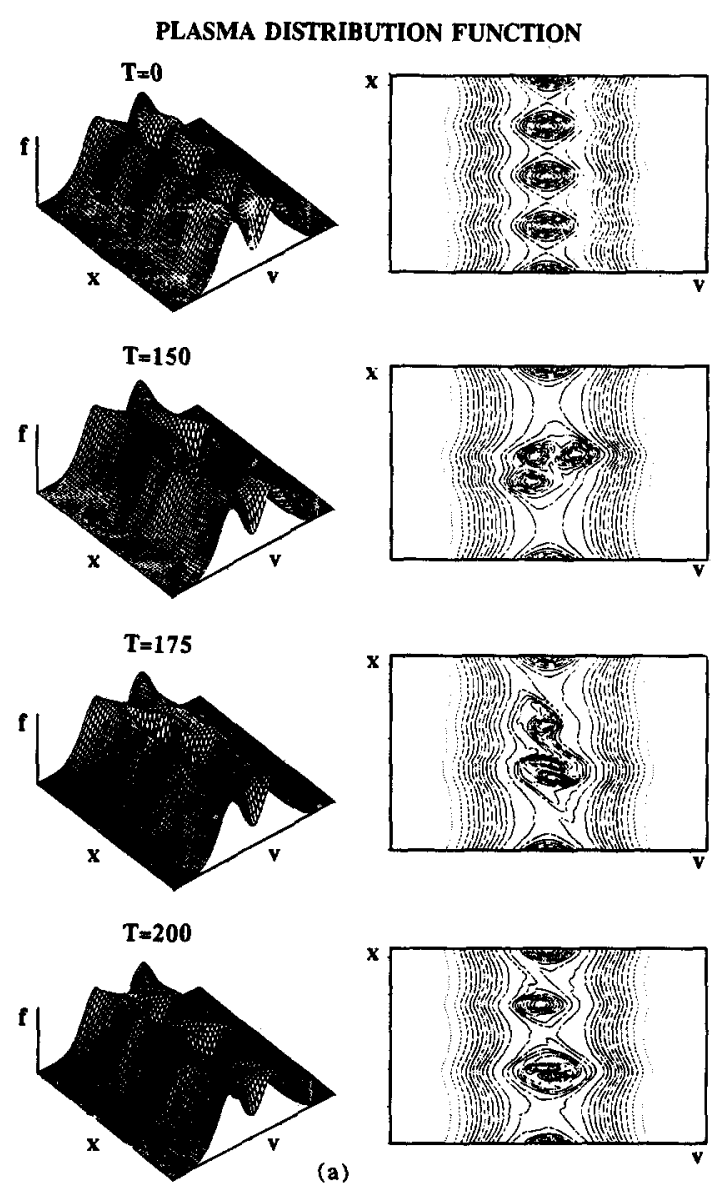

(a)
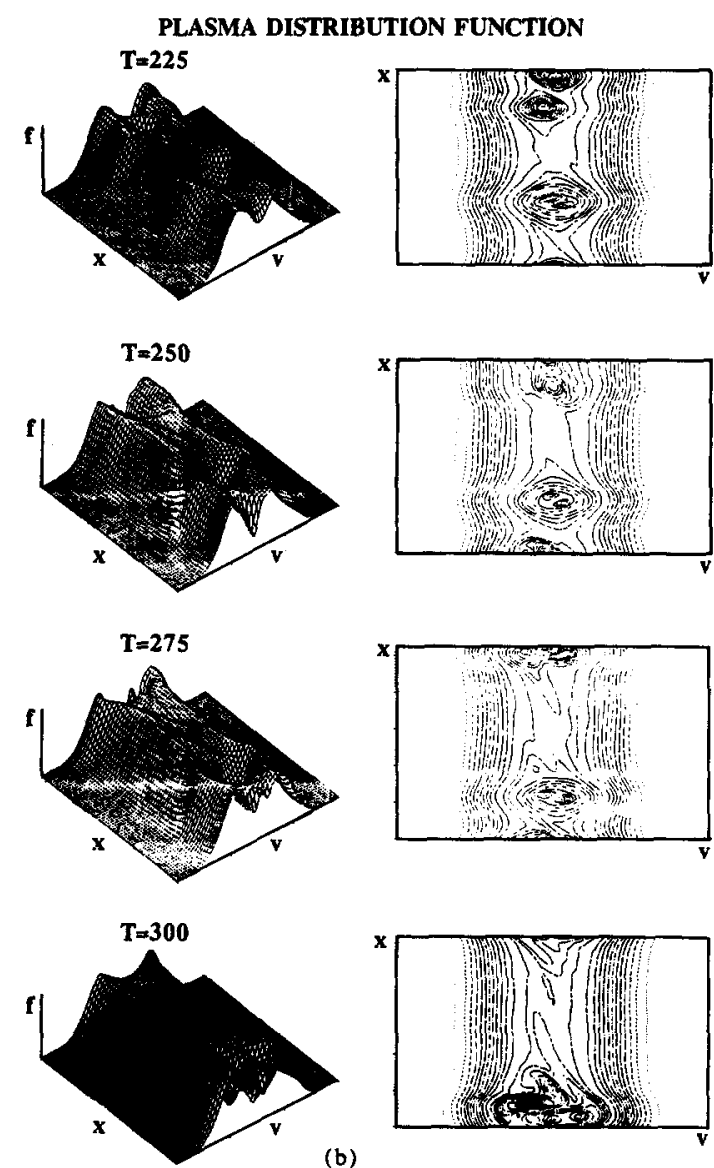

(b)
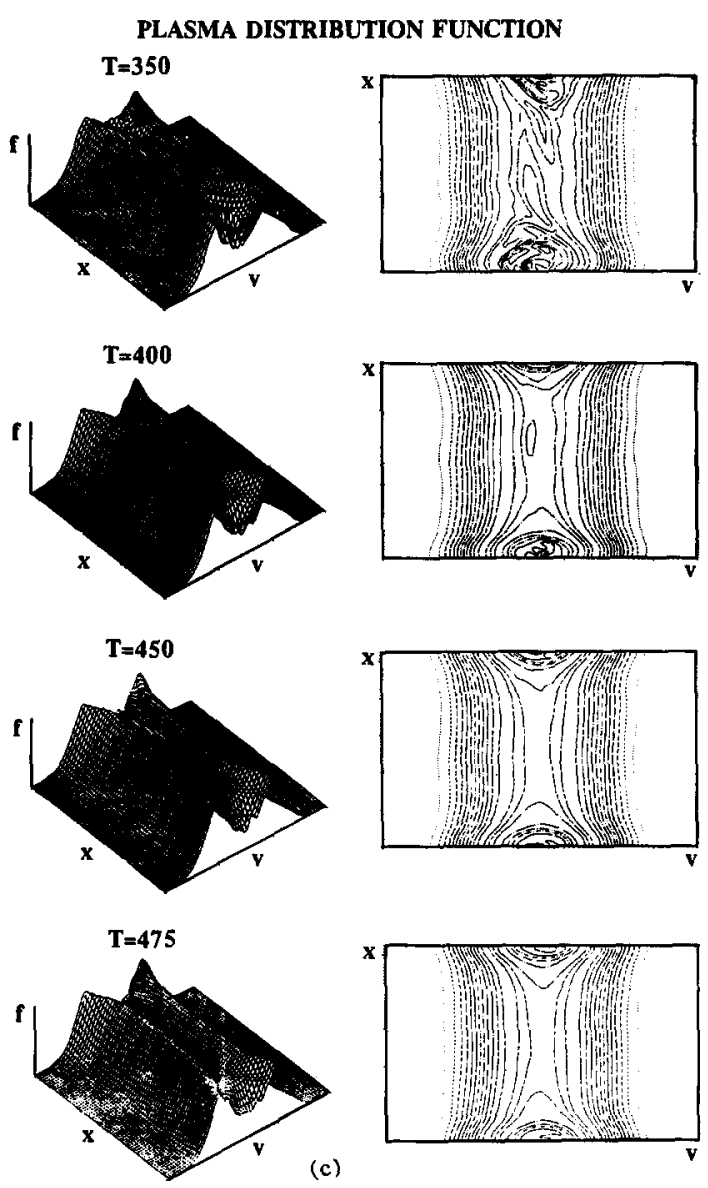

(c) 

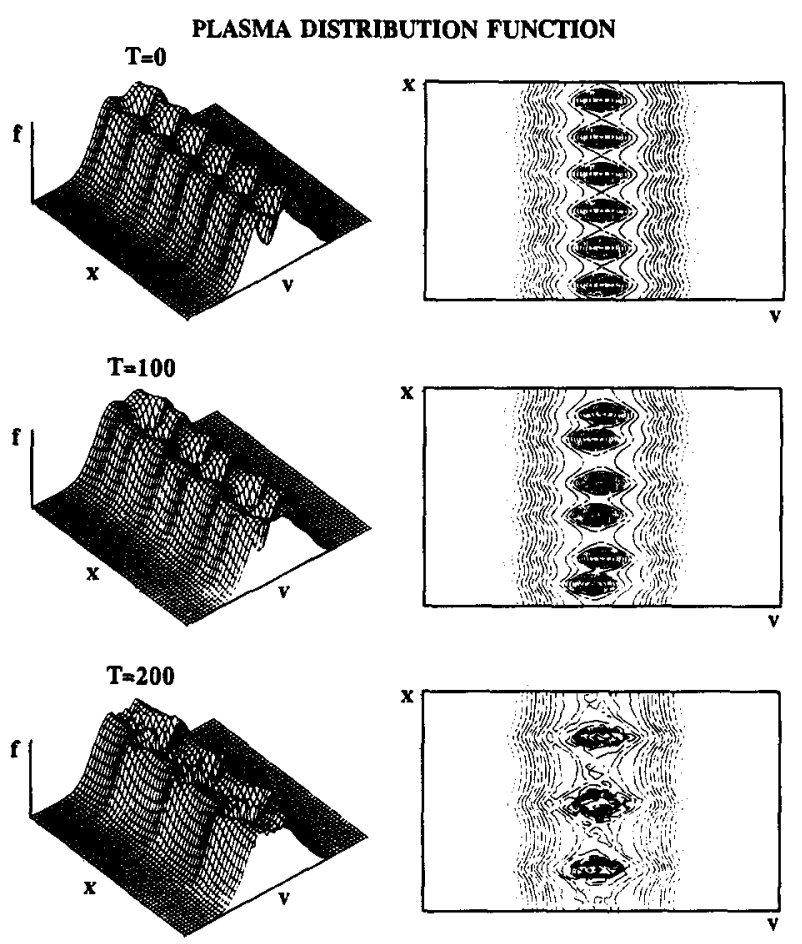

(a)
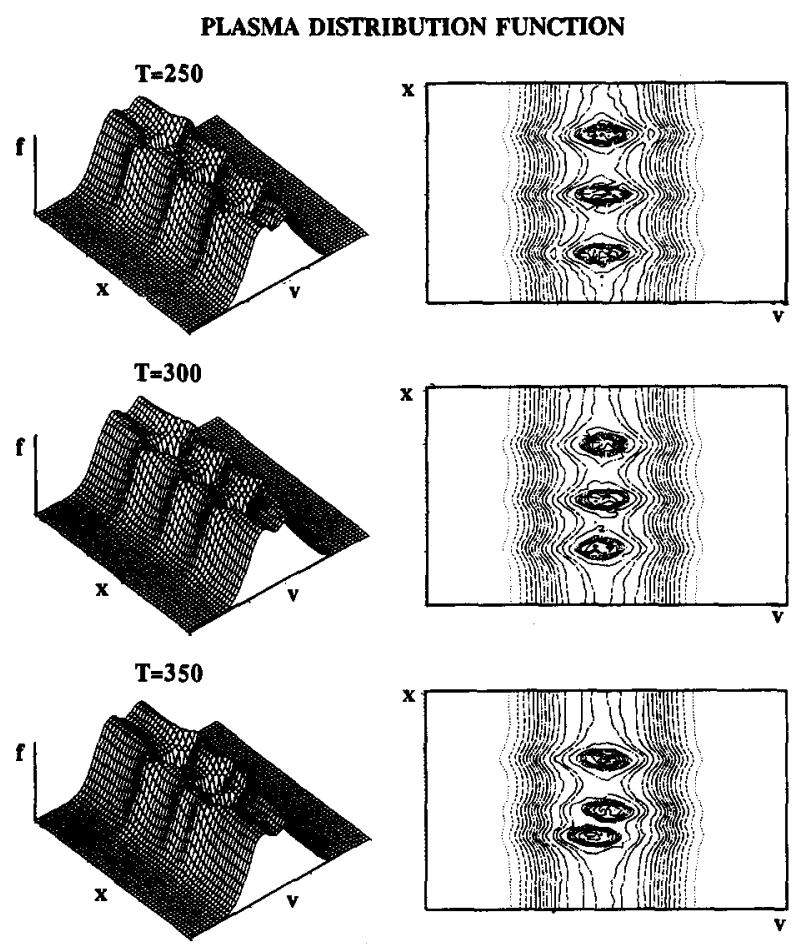

(b)

FIG. 11. The same as Fig. 7 for $N=6$ holes.

length of the system and then their coalescence into a onehole structure. An instructive movie of the phenomena has been produced showing a three-hole case.

\section{COMMENTS AND CONCLUSION}

The use of noiseless extremely stable Eulerian codes on a vector computer allows us to obtain precise information on
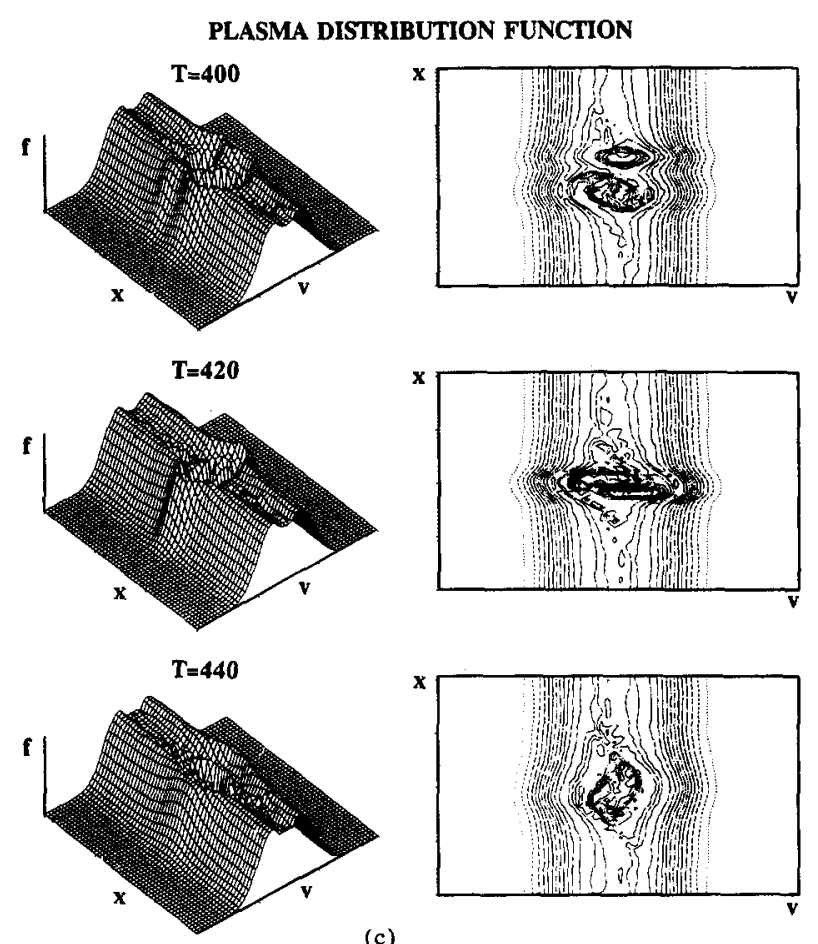

(c)
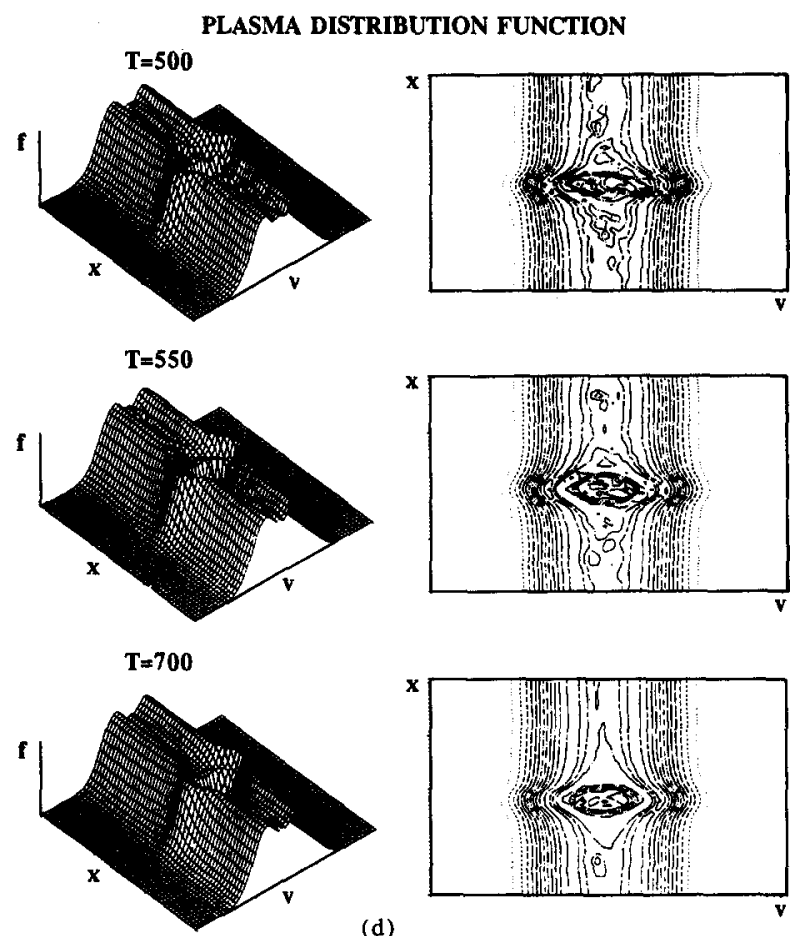

(d)

phase space behavior of a one-dimensional electron Vlasov plasma, over a long time, with a large spectrum of wavenumbers.

The BGK structures with holes (vortices) in phase space have been found to be unstable, although they first appear at the initial stage of a two-stream homogeneous plasma. But in all cases the interaction of the vortices brings a coalescence until only one subsists. In fact, the stabilization 

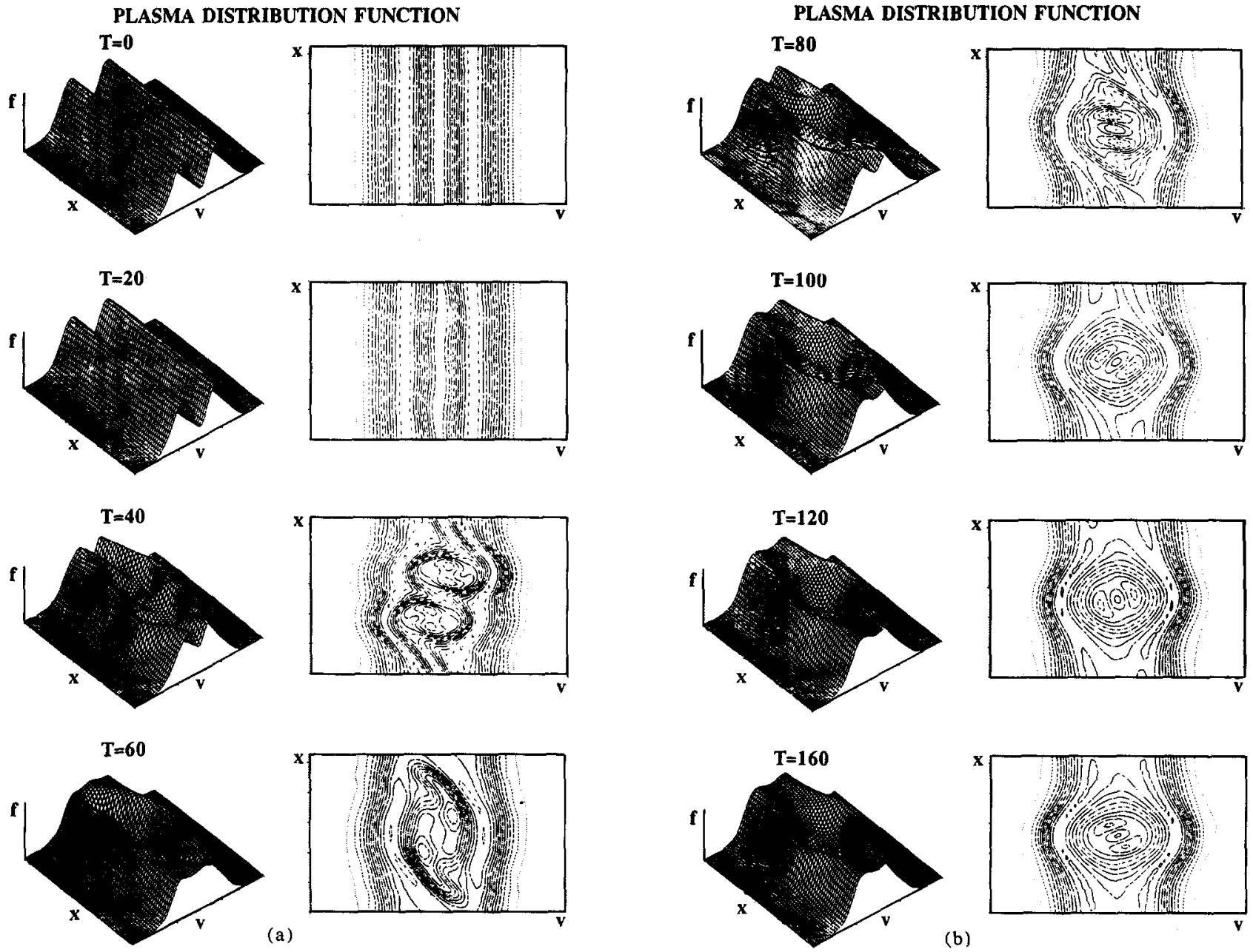

FIG. 12. Evolution of the two-stream homogeneous plasma in a periodic box of length $L=30.78$, which is twice the marginal wavelength of 15.39 . Note the appearance of a two-hole structure at $\omega_{p} t=40$ and its subsequent coalescence.

is related to the finite size of the system, since a system of one period is indeed marginally stable. This result is in agreement with a theory predicting an evolution of energy toward equilibrium structures of long wavelengths ${ }^{10}$ for a two-dimensional system (either fluid or phase space). The stability of a one-hole system is also consistent with entropy arguments explaining the tendency of holes to coalesce. ${ }^{11}$

Two points must be made.

(i) The first point concerns the rather smooth structure in the final state in phase space. Although the results presented here have been obtained with the Fourier code, they are identical with those obtained with the cubic spline code. Nevertheless, an examination of the long time behavior (up to $2700 \omega_{p}^{-1}$ ) shows that it takes somewhat longer to obtain the "cleaning" of the microstructure with the spline code and is related to the treatment of the small wavelengths, which is numerically quite different. However, both codes exhibit, after the "cleaning," the same final vortex with a distribution function that is shown to be a function of the sole energy. Further physical and numerical studies should be made of the precise mechanism of this "cleaning."

(ii) The second interesting feature is the tendency of holes to behave as macroparticles ${ }^{21}$ before coalescence. The point has been studied by considering different values of the phase $\psi$ in the initial perturbation (22) on a $N=2$ system. Taking $\psi \neq 0$ brings a coalescence of the two vortices in a time depending on $\psi$. However, by choosing $\psi=0$ no evolution can be noticed. This last case corresponds to a perfect symmetry in the system, so that the "forces" acting on the holes balance one another perfectly. Thus a small breaking in the symmetry can create an imbalance of the forces acting upon each hole. For more details, see Ref. 21. Furthermore, we have also studied the time evolution of the position of the center of mass of each vortex, confirming the existence of "attractive" forces between vortices, depending only on the distance. The existence of these attractive forces was mentioned for the first time by Berk, Nielson, and Roberts ${ }^{8}$ using a water bag model. The present Eulerian codes specify some properties of these forces. The study of the inelastic character of the collision between holes is now under consideration.

Finally, we note that holes in phase space are closely related to the "clumps" that appear in weak turbulence theory, ${ }^{11}$ although BGK instabilities give rise to strongly nonlinear effects. 


\section{ACKNOWLEDGMENTS}

This research was made possible through the allocation of computer time on the CRAY-1 machine of the "Centre de Calcul Vectoriel pour la Recherche," and graphic facilities of the CIRCE.

The authors are also indebted to the Association France-Québec (Grant No. 20090186 ) for the support during the research.

${ }^{1}$ I. Bernstein, J. Greene, and M. D. Kruskal, Phys. Rev. 108, 546 (1957). ${ }^{2}$ F. Hohl, Phys. Fluids 12, 230 (1969).

${ }^{3}$ J. P. Doremus, U. Finzi, J. Holec, and M. R. Feix, Plasma Phys. 16, 189 (1974).

${ }^{4}$ M. R. Feix, in Proceedings of the Culham Symposium of Turbulence and Nonlinear Effects in Plasma, edited by B. E. Keen and E. W. Laing (Culham Laboratory, Abingdon, England, 1973), pp. 139-183.

${ }^{5}$ H. R. Lewis and K. R. Symon, J. Math. Phys. 20, 413 (1979).
6J. L. Schwarzmeier, H. R. Lewis, B. Abraham-Shrauner, and K. R. Symon, Phys. Fluids 22, 1747 (1979).

${ }^{7}$ K. V. Roberts and H. L. Berk, Phys. Fluids 10, 1595 (1967).

${ }^{8}$ H. L. Berk, C. E. Nielsen, and K. V. Roberts, Phys. Fluids 13, 980 (1970).

${ }^{9}$ U. Finzi, Plasma Phys. 14, 327 (1972).

${ }^{10}$ G. Knorr, Plasma Phys. 19, 529 (1977).

${ }^{11}$ T. M. Dupree, Phys. Fluids 25, 277 (1982).

${ }^{12}$ T. Boutros-Ghali and T. M. Dupree, Phys. Fluids 24, 1839 (1981).

${ }^{13}$ M. V. Goldman, Phys. Fluids 13, 1281 (1970).

${ }^{14}$ E. Minardi, Plasma Phys. 14, 427 (1972).

${ }^{15}$ P. Bertrand, J. P. Doremus, G. Baumann, and M. R. Feix, Phys. Fluids 15, 1275 (1972).

${ }^{16} \mathrm{~F}$. Santini, Plasma Phys. 12, 165 (1970).

${ }^{17}$ G. Knorr and C. G. Cheng, J. Comput. Phys. 22, 330 (1976).

${ }^{18}$ R. J. Gagne and M. M. Shoucri, J. Comput. Phys. 24, 445 (1977).

${ }^{19}$ M. M. Shoucri and R. J. Gagne, J. Comput. Phys. 27, 315 (1978).

${ }^{20}$ A. Ghizzo, B. Izrar, P. Bertrand, M. R. Feix, E. Fijalkow, and M. M. Shoucri, in Proceedings of the 8th Europhysics Conference on Computational Physics (European Physical Society, Budapest, 1986), Vol. 10 D, pp. 109-112.

${ }^{21}$ A. Ghizzo, B. Izrar, P. Bertrand, M. R. Feix, E. Fijalkow, and M. M. Shoucri, Phys. Lett. A 120, 191 (1987). 